\title{
Biliary Tract Cancer: A Survey Regarding the Current Oncological Daily Care Practice in Germany
}

\author{
Marianne Sinn ${ }^{\mathrm{a}} \quad$ Sven Bischoff $^{\mathrm{a}}$ Oliver Nehls ${ }^{\mathrm{b}} \quad$ Uwe Pelzer $^{\mathrm{a}} \quad$ Fritz von Weizsäcker $^{\mathrm{c}}$ \\ Stefan Kubicka $^{d}$ Jens M. Stieler ${ }^{\mathrm{a}}$ Karel Caca $^{\mathrm{e}}$ Hanno Riess $^{\mathrm{a}}$
}

a Medizinische Klinik mit Schwerpunkt Hämatologie und Onkologie, Charité - Universitätsmedizin Berlin,

${ }^{\mathrm{b}}$ Gastroenterologie und Tumormedizin an St. Anna, Stuttgart,

'Innere Medizin I, Schlosspark-Klinik, Berlin,

dMedizinische Klinik I, Klinikum am Steigenberg Reutlingen,

${ }^{e}$ Klinik für Innere Medizin, Gastroenterologie, Hämato-Onkologie, Diabetologie und Infektiologie, Klinikum Ludwigsburg, Germany

\section{Keywords}

Biliary tract cancer - Survey · Oncologic daily care practice

\section{Summary}

Background: The low incidence and the variable presentation complicate clinical investigations on biliary tract cancer. The results of Valle et al. in 2009 provided, for the first time, an evidence-based palliative treatment for this rare tumor type. So far no data are available in Germany regarding the current daily care practice. Methods: We started this national survey in May 2011, including about 3,400 members of the AIO (Working Group Medical Oncology), DGHO (German Society of Hematology and Oncology) and GGHBB (Society of Gastroenterology and Hepatology in Berlin and Brandenburg). The standardized online form contained questions concerning field of action and diagnostic and therapeutic procedures. Evaluation was conducted anonymously. Results: 162 responses could be obtained, corresponding to a response rate of about $5 \% .70 .4 \%$ of the respondents were physicians in hospitals, $23.5 \%$ stated to work in private practices. $61.7 \%$ of the respondents were medical oncologists and $27.2 \%$ gastroenterologists. $52.5 \%$ of the participants pointed out to use the standard regimen of gemcitabine and cisplatin. For second-line regimen, the most frequent answer (29\%) specified the administration of oxaliplatin in combination with 5-fluorouracil (5-FU) or capecitabine. Conclusions: This survey may help to clarify the current oncologic daily care procedures for patients with biliary tract cancer in Germany. The results can be helpful for further clinical investigations or the implementation of a tumor-specific register.

\author{
Schlüsselwörter \\ Karzinome der Gallenwege · Umfrage · \\ Versorgungswirklichkeit
}

\section{Zusammenfassung}

Hintergrund: Die niedrige Inzidenz sowie die uneinheitliche klinische Darstellung erschweren die onkologische Forschung bei Karzinomen der Gallenwege. Erst seit 2009 steht mit den Ergebnissen von Valle et al. eine evidenzbasierte palliative Chemotherapie für diese seltene Tumorerkrankung zur Verfügung. Es existieren keine Daten über die aktuelle Versorgungssituation in Deutschland. Methoden: In dieser Umfrage wurden alle der etwa 3400 Mitglieder der Arbeitsgemeinschaft Internistische Onkologie (AIO), der Deutschen Gesellschaft für Hämatologie und Onkologie (DGHO) und der Gesellschaft für Gastroenterologie und Hepatologie in Berlin und Brandenburg (GGHBB) befragt. Das standardisierte Online-Formular beinhaltete Fragen zum Arbeitsumfeld der Teilnehmer sowie zu angewendeten diagnostischen und therapeutischen Maßnahmen. Die Auswertung erfolgte anonymisiert. Ergebnisse: 162 Fragebogen wurden beantwortet (Rücklaufquote 5\%). 70,4\% der Teilnehmer gaben an, in Krankenhäusern tätig zu sein, 23,5\% kamen aus dem niedergelassenen Bereich. Die Teilnehmer waren zu 61,7\% internistische Onkologen und zu 27,2\% Gastroenterologen. Nur etwa die Hälfte $(52,5 \%)$ setzen in der palliativen Erstlinien-Behandlung den aktuellen Therapiestandard mit Gemcitabin/Cisplatin ein. In der ZweitlinienTherapie wurde am häufigsten (von 29\% der Befragten) die Anwendung von Oxaliplatin in Kombination mit 5-Fluoruracil (5-FU) oder Capecitabin angegeben. Schlussfolgerungen: Diese Umfrage trägt dazu bei, die aktuelle Versorgungssituation in Deutschland für Patienten mit Karzinomen der Gallenwege darzustellen. Die Ergebnisse können für weitere klinische Forschungen ebenso hilfreich sein wie für den Aufbau eines tumorspezifischen Registers.

Dr. med. Marianne Sinn

Charité - Universitätsmedizin Berlin, Medizinische Klinik

mit Schwerpunkt Hämatologie und Onkologie

Campus Virchow Klinikum

Augustenburger Platz 1, 13353 Berlin, Germany

marianne.sinn@charite.de 


\section{Introduction}

Biliary tract cancer (BTC) is an uncommon but challenging tumor entity requiring multidisciplinary engagement. The anatomic conditions of the tumors with possible origin from intra- or extrahepatic bile ducts or the gall bladder can lead to difficulties in the diagnostic and therapeutic management. Teamwork between surgeons, gastroenterologists, internal oncologists and radiologists is necessary to ensure an optimal result in patient-centered care.

The low incidence, the variety of presentation, and the sometimes confusing heterogeneity in nomenclature complicate clinical investigations and treatment recommendations. In the last decades, many phase II studies were performed trying to define a standard of care for palliative chemotherapy [1], until no more than two years ago the combination of gemcitabine and cisplatin was established as first-line chemotherapy in advanced BTC [2]. The current European Society for Medical Oncology (ESMO) guideline for biliary cancer [3] adopted this therapeutic regimen as recommendation for first-line chemotherapy, but today in Germany no evidencebased (S2 or S3) guideline for BTC is available (AWMF online; Arbeitsgemeinschaft der Wissenschaftlichen Medizinischen Fachgesellschaften, Working Group of the Scientific Medical Associations). The aim of our project was to evaluate the implementation of a new evidence-based therapy in clinical practice and to detect differences and similarities in the diagnostic and therapy modalities in dependence on the working environment (subspecialization and clinical environment) of the participating physicians.

What kind of diagnostic and therapeutic procedures were performed and what are the differences between and the similarities of the different engaged disciplines or between hospital and private practice? How are the results from clinical research transported into clinical reality?

\section{Methods}

We started this self-administered national survey in May 2011, including questions concerning the field of action of the participating physicians and their applied diagnostic and therapeutic procedures. The questions were discussed and determined in the 'Leitgruppe hepatobiliäre Tumore' of the AIO (Arbeitsgemeinschaft Internistische Onkologie, Working Group Medical Oncology). The standardized online form was sent via e-mail to all (about 3,400 members) of the AIO, the DGHO (Deutsche Gesellschaft für Hämatologie und Onkologie, German Society of Hematology and Oncology) and the GGHBB (Gesellschaft für Gastroenterologie und Hepatologie in Berlin und Brandenburg, Society of Gastroenterology and Hepatology in Berlin and Brandenburg). The contacted physicians were not preselected. E-mail addresses were filtered to avoid double participation. Respondents submitted their responses online; participation and evaluation were done anonymously. The database was closed in September 2011.

Initially, the participants were asked how much patients with biliary tract cancer (subdivided into intrahepatic, extrahepatic, and gallbladder origin) were treated per year. Concerning the field of action, data on the subspecialization (medical oncology, gastroenterology, surgery, radio- therapy, or other) and the field of action (university hospital, maximum care hospital, standard care hospital, private practice, or other) were acquired. Further questions were related to neoadjuvant, adjuvant, and palliative treatments in general as well as the cytotoxic agents and regimens used in first- or second-line chemotherapy. The participants were asked to differentiate between locally advanced and metastatic disease. A final question asked for an evaluation of a disease-specific register.

\section{Statistical Analysis}

CKForms 1.3.5 (a Joomla content management system component) software was used for the administration of the survey. There was no possibility of tracking the participants.

Descriptive tests and statistical analysis were performed to collect and to compare the given answers between field of action/specialty subgroups using the Statistical Package for the Social Sciences (SPSS; German version SPSS 19.0) and Microsoft Excel 2003. Predefined subgroup analysis was performed by grouping the results of each question with regard to subspecialization.

\section{Results}

\section{Participants}

Between May 2011 and September 2011, 162 physicians took part in the survey, resulting in a response rate of approximately $5 \%$.

More than two-thirds of the respondents $(70.4 \% ; \mathrm{n}=114)$ are physicians in hospitals (university hospital $>$ hospital of standard care $>$ hospital of maximum care). About one-fourth of the respondents $(23.5 \% ; \mathrm{n}=38)$ are working in private practices. The majority of the participants are medical oncologists $(61.7 \% ; n=100)$, about one-third of them $(27.2 \%$; $\mathrm{n}=44)$ are gastroenterologists, and the remainder are surgeons $(6.8 \% ; n=11)$ or radiation therapists $(1.2 \% ; n=2)$. More than half of the participating physicians stated to treat more than 10 patients per year (11-25 patients $32.7 \% ; \mathrm{n}=53$; more than 25 patients $24.7 \% ; \mathrm{n}=40)$. About one-fifth of the participants answered to treat 10 patients or less (6-10 patients $16.7 \% ; \mathrm{n}=27 ;<5$ patients $5.6 \% ; \mathrm{n}=9$ ).

For details, please see figures 1 and 2 .

\section{Diagnostic Procedures}

Most respondents considered histological confirmation $(75.9 \% ; \mathrm{n}=123)$, evaluation of tumor markers $(69.8 \%$; $\mathrm{n}=113)$, radiological assessments with computed tomography (CT) or magnetic resonance imaging (MRI) $(85.2 \% ; n=138)$ or ultrasound $(72.8 \% ; \mathrm{n}=118)$, and endoscopic evaluation with endoscopic retrograde cholangiopancreatography (ERCP) or endosonography $(79 \% ; n=128)$ as part of the confirmation diagnostic management.

CT or MRI scans as diagnostic procedure for tumor assessment in the course of disease was mentioned in the majority of cases $(77.8 \% ; \mathrm{n}=126)$, followed by abdominal ultrasound $(73.5 \% ; \mathrm{n}=119)$ and determination of tumor markers $(68.5 \%$; 

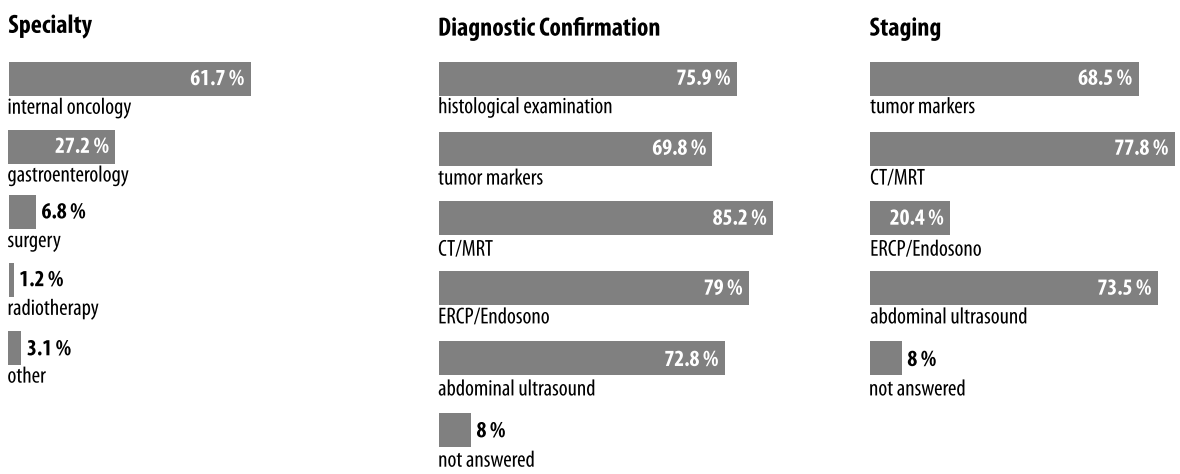

Fig. 1. Subspecialization and diagnostic procedures.

Fig. 2. Participants" characteristics.
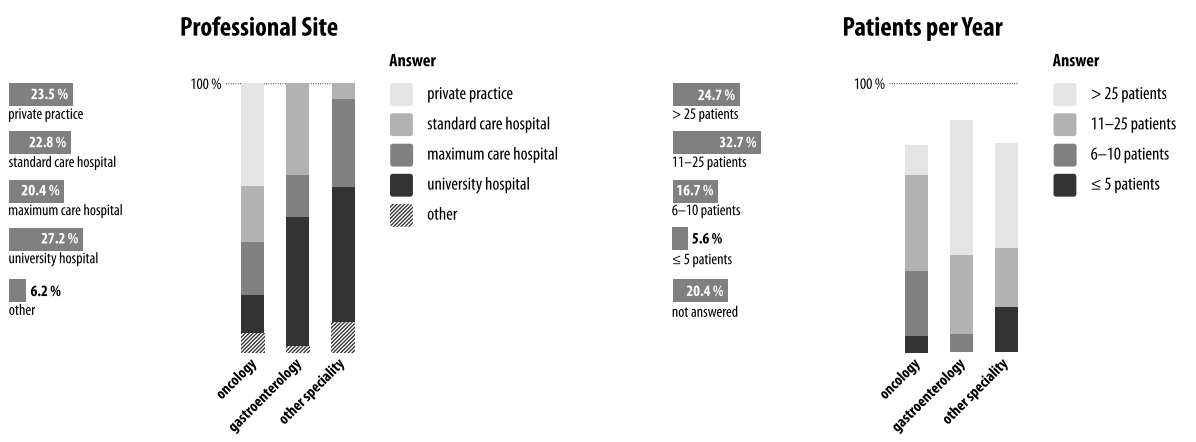

$\mathrm{n}=111)$. Endoscopy was named by about one-fifth of the participants $(20.4 \% ; \mathrm{n}=33)$.

The majority $(79.6 \% ; n=129)$ considered a follow-up period of 3 months as reasonable; only a few participants $(7.4 \% ; n=12)$ voted for every 6 months. A more frequent follow-up of every $4-8$ weeks was mentioned by a small subgroup $(3.1 \% ; n=5)$, and only some participants $(5.6 \% ; n=9)$ stated not to do any follow-up at all (fig. 4).

\section{Therapy}

\section{Neoadjuvant}

Only a small number of participants stated to perform neoadjuvant treatment as routine procedure $(3.1 \% ; n=5)$. About one-third of the participants $(29 \% ; n=47)$ considered this as a therapeutic option for patients who seemed to be not safely operable. For about two-thirds of the participants $(63 \% ; n=102)$ neoadjuvant treatment was no therapeutic option, independently of preexisting conditions.

\section{Adjuvant}

Concerning adjuvant therapy, different treatment options were inquired.

Adjuvant chemotherapy was chosen by about 1 out of 5 participants $(19.8 \% ; \mathrm{n}=32)$, and about 1 out of 4 participants $(27.8 \% ; n=45)$ mentioned to consider this option if risk factors such as R1 resection status, positive lymph nodes, or elevated postoperative carbohydrate antigen (CA) 19-9 levels were present. Adjuvant radiochemotherapy or radiotherapy was chosen by only a small subgroup $(1.2 \%$ or $3.1 \%$, respec- tively; $\mathrm{n}=2$ or 5 ). This number increased to about 1 out of 5 respondents $(19.1 \%$ or $15.4 \%$, respectively; $\mathrm{n}=31$ or 25$)$ if one of the named risk factors was present.

\section{Palliative}

For the palliative treatment of locally advanced disease, the majority of the respondents stated to apply chemotherapy $(87 \% ; n=141)$, about one-fifth each of the participants chose resection $(22.2 \% ; \mathrm{n}=36)$ or radiotherapy $(25.9 \% ; \mathrm{n}=42)$. Multiple responses were allowed.

Asked for cytotoxic regimens in first-line therapy, approximately half $(52.5 \% ; \mathrm{n}=85)$ of the participants pointed out to use the standard regimen of gemcitabine and cisplatin. About 1 out of 4 participants $(24.1 \% ; n=39)$ indicated the use of gemcitabine in combination with oxaliplatin, and about 1 out of 5 participants $(21.6 \%$; $n=35)$ stated to apply gemcitabine alone. Less than $5 \%$ of the participants $(4.3 \% ; n=7)$ answered to use other regimens.

With regard to second-line regimens, the most frequent answer $(29 \% ; n=47)$ was the administration of oxaliplatin in combination with 5-fluorouracil (5-FU) or capecitabine. 5-FU or capecitabine alone $(25.3 \% ; n=41)$ was named at an almost equal frequency. The use of mitomycin $\mathrm{C}$ or taxane was stated by less than $5 \%$ of the participants $(3.1 \%$ or $2.5 \% ; n=5$ or 4 , respectively).

Regarding third-line therapy, the responding physicians most frequently named mitomycin $\mathrm{C}$ alone or in combination $(14.2 \% ; n=23)$, followed by taxane $(8.6 \% ; n=14)$. A small subgroup $(3.7 \% ; n=6)$ stated to not consider any third-line therapy at all.

For details, please see figures 3 and 4 . 


\begin{tabular}{|c|c|c|}
\hline Neoadjuvant Therapy & Adjuvant Radiotherapy & Adjuvant Chemotherapy \\
\hline $3.1 \%$ & $3.1 \%$ & $19.8 \%$ \\
\hline & yes & yes \\
\hline $29 \%$ & $15.4 \%$ & $27.8 \%$ \\
\hline only if not certainly operable & only if risk factors present & only if risk factors present \\
\hline $63 \%$ & $77.2 \%$ & $46.9 \%$ \\
\hline no & no & no \\
\hline $4.9 \%$ & $4.3 \%$ & $5.6 \%$ \\
\hline not answered & not answered & not answered \\
\hline Adjuvant Radiochemotherapy & Treatment of Locally Advanced Stages & \\
\hline | $1.2 \%$ & $87 \%$ & \\
\hline yes & chemotherapy & \\
\hline $19.1 \%$ & $22.2 \%$ & \\
\hline only if risk factors present & resection & \\
\hline $74.7 \%$ & $25.9 \%$ & \\
\hline no & radiotherapy & \\
\hline $4.9 \%$ & $6.8 \%$ & \\
\hline not answered & not answered & \\
\hline Firstline Regimen & Secondline Regimen & Thirdline Regimen \\
\hline $52.5 \%$ & $25.3 \%$ & $14.2 \%$ \\
\hline Gemcitabine//isplatin & 5-FU/Capecitabine mono & Mitomycin \pm combination \\
\hline $24.1 \%$ & $29 \%$ & $8.6 \%$ \\
\hline Gemcitabine/0xaliplatin & Oxaliplatin + 5-FU/Capecitabine & Taxane \\
\hline $21.6 \%$ & $3.1 \%$ & $3.7 \%$ \\
\hline Gemcitabine mono & Mitomycin & none \\
\hline $\begin{array}{l}4.3 \% \\
\text { other }\end{array}$ & $\underset{\text { Taxane }}{2.5 \%}$ & \\
\hline $14.2 \%$ & & \\
\hline
\end{tabular}

Fig. 3. Considered treatment options and applied regimens.

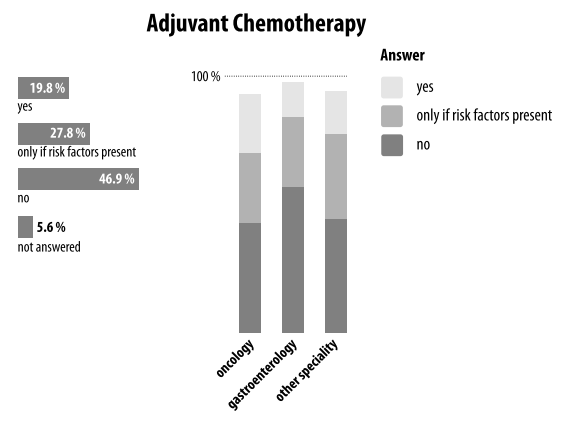

Firstline Regimen

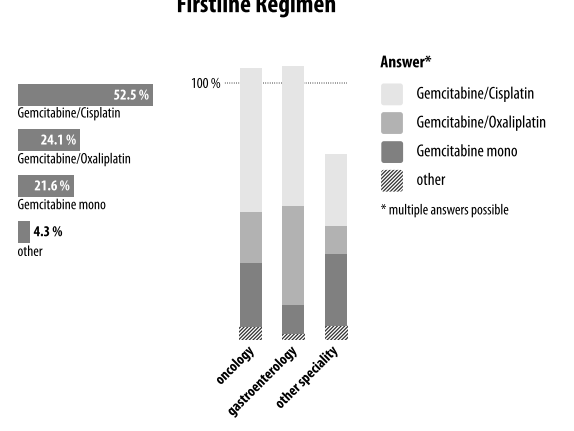

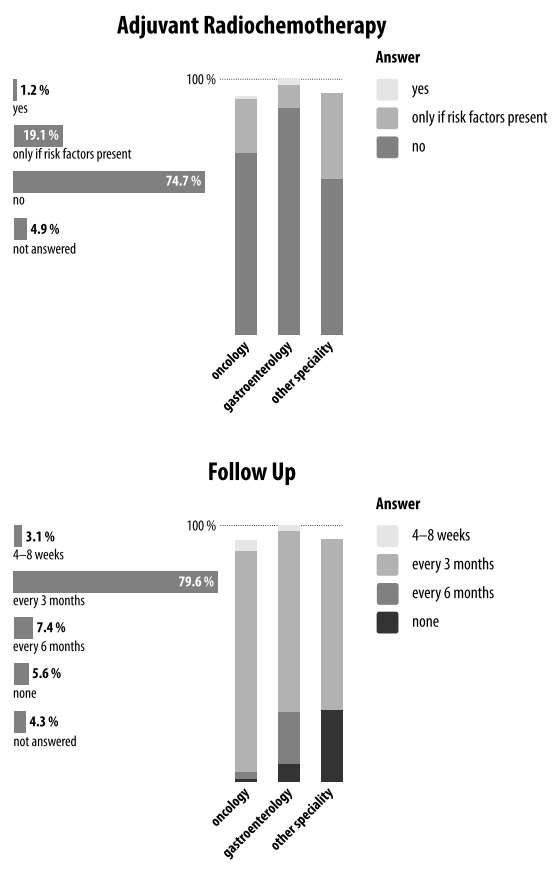

Fig. 4. Treatment options and follow-up interval by subspecialization.

Sinn/Bischoff/Nehls/Pelzer/von Weizsäcker/ Kubicka/Stieler/Caca/Riess 


\section{Analysis of Subgroups}

\section{Field of Action and Number of Patients}

Participants working in university hospitals stated to treat predominantly more than 25 patients per year $(54.5 \%$; $\mathrm{n}=24)$. About half of the participants of standard care hospitals $(43.2 \% ; n=16)$ and of maximum care hospitals $(36.4 \%$; $\mathrm{n}=12$ ) declared to take care of 11-25 patients per year, about one-fifth of both indicated to treat more than 25 patients per year (maximum care hospital $21.2 \%$; $n=7$; standard care hospital $18.9 \% ; n=7)$. Few of the respondents from private practices $(2.6 \% ; n=1)$ treat more than 25 patients per year, most of them stating to treat more than 5 patients per year $(6-10$ patients $36.8 \%$; $\mathrm{n}=14 ; 11-25$ patients $31.6 \%$; $\mathrm{n}=12)$.

\section{Specialization}

Significant subgroups were oncologists with $61.7 \%(\mathrm{n}=100)$ and gastroenterologists with $27.2 \%(n=44)$ of the participants. The remaining physicians were surgeons $(6.8 \%$; $\mathrm{n}=11)$, radiation therapists $(1.2 \% ; \mathrm{n}=2)$, and others $(3.1 \%$; $\mathrm{n}=5$ ), in the following subsumed in a single subgroup representing about $10 \%$ of the participants. In the analysis, mainly oncologists and gastroenterologists were compared.

Concerning procedures in the primary diagnostic process, more gastroenterologists stated to initiate histological confirmation $(86.4 \%$ vs. $77.0 \%$ oncologists; $\mathrm{n}=38$ vs. 77$), \mathrm{CT} / \mathrm{MRI}$ $(90.9 \%$ vs. $84.0 \% ; n=40$ vs. 84$)$, abdominal ultrasound (79.5\% vs. $74.0 \% ; \mathrm{n}=35$ vs. 74$)$, and endoscopic interventions ( $86.4 \%$ vs. $77.0 \%$; $n=38$ vs. 77 ). Tumor markers were appointed by $73 \%(n=73)$ of oncologists and $63.6 \%(n=28)$ of gastroenterologists. 3 months was considered by most of the respondents $(70.5 \%, \mathrm{n}=31$ gastroenterologists; $86 \%, \mathrm{n}=86$ oncologists) as reasonable interval for follow-up investigations, but more gastroenterologists chose 6 months $(20.5 \%$, $\mathrm{n}=9$ vs. $3.0 \%, \mathrm{n}=3$ oncologists $)$ or no follow-up at all $(6.8 \%$, $\mathrm{n}=3$ vs. $1.0 \%, \mathrm{n}=1)$.

With regard to neoadjuvant or adjuvant management, only a few gastroenterologists $(2.3 \% ; n=1)$ and oncologists $(3 \%$; $\mathrm{n}=3$ ) apply neoadjuvant treatment. This was increased to about 1 out of 3 (29.5\%; $\mathrm{n}=13$ gastroenterologists/29.0\%; $\mathrm{n}=29$ oncologists) if a patient is not safely operable. The general use of an adjuvant radiation therapy was indicated by less than $5 \%(2.3 \%, \mathrm{n}=1$ gastroenterologist; $3.0 \%, \mathrm{n}=3$ oncologists).

Adjuvant radiochemotherapy in the presence of risk factors (R1 resection, positive lymph nodes, elevated CA 19-9 levels) was named by every fifth oncologist $(21.0 \% ; n=21)$ and about every tenth gastroenterologist $(9.1 \% ; n=4)$. Adjuvant chemotherapy alone was quoted by about 1 out of 4 oncologists $(23.0 \% ; \mathrm{n}=23)$ and 1 out of $8(13.6 \% ; \mathrm{n}=6)$ gastroenterologists. If risk factors were presumed, about every fifth respondent indicated to use adjuvant chemotherapy (gastroenterologists $27.3 \%, \mathrm{n}=12$; oncologists $27.0 \%, \mathrm{n}=27$ ).
The use of palliative chemotherapy was named by the majority of oncologists $(88.0 \% ; \mathrm{n}=88)$ and gastroenterologists $(90.9 \% ; n=40)$; about 1 out of 5 respondents specified the employment of palliative radiation therapy (gastroenterologists $22.7 \%, \mathrm{n}=10$; oncologists $26.0 \%, \mathrm{n}=26$ ).

For first-line therapy, the application of the combination of gemcitabine and cisplatin was indicated almost equally by $54.5 \%(n=24)$ of the participant gastroenterologists and by $56.0 \%(\mathrm{n}=56)$ of the oncologists. However, more gastroenterologists indicated the use of the combination of gemcitabine and oxaliplatin $(38.6 \%, \mathrm{n}=17$ gastroenterologists vs. $20.0 \%, \mathrm{n}=20$ oncologists), and more oncologists named the application of gemcitabine alone $(25.0 \%, \mathrm{n}=25$ oncologists vs. $11.4 \%, \mathrm{n}=5$ gastroenterologists) (fig. 4 ).

\section{Register}

Asked for a tumor-specific register, almost 90\% ( $\mathrm{n}=143)$ of the participants voted for the introduction, and about half of them affirmed an active cooperation. Less than 10\% (7.4\%; $=12$ ) indicated to estimate the implementation as not useful.

\section{Discussion}

Our survey was sent to all members of the AIO and DGHO, both organizations predominantly representing physicians specialized in medical oncology. GHBB as a local BerlinBrandenburg society with a focus on gastroenterology was included to strengthen the position of the gastroenterologists. With approximately $5 \%$, the response rate was low, and even a little lower than in preceding German surveys concerning oncologic themes such as the current oncologic treatment of patients with pancreatic cancer in Germany [4] and the treatment of epidermal growth factor receptor antagonist-induced skin rash [5]. This is most likely due to the fact that only a very small number of specialized physicians in Germany is actively engaged in the treatment of patients with BTC.

A survey regarding patient-centered care in BTC should first of all answer the question whether the state of the art is implemented in everyday patient care and then analyze exemplarily if and how new and evidence-based results from clinical research were transferred into real-life practice. In the last decades, many phase II studies - generally with few included patients - were conducted for patients with BTC, but only in 2008 the results of the phase III study of Valle et al. [2] became available, introducing a standard of care for palliative chemotherapy. The results were presented for the first time at the annual meeting of the American Society of Clinical Oncology (ASCO), one of the main opinion-forming events in oncology, and the data became widely available in 2009 when published in the New England Journal of Medicine.

How is such a new standard of care transported from relevant publishing organs to oncologic 'reality'? No German guidelines for BTC exist or are in preparation at the moment; 
however, in the actual guidelines of the ESMO concerning BTC, this therapeutic regime was approved [3]. In our survey, about half of the respondents stated to use the combination of gemcitabine and cisplatin as first-line chemotherapy, but the remaining respondents declared to apply other regimes. Considering that the participants were physicians interested in BTC, it seems questionable why the new therapeutic standard is not adopted to a greater extent. Similar results were published recently for Dutch hospitals where the guideline adherence for antithrombotic therapy in acute coronary syndrome was quite diverse [6]. Few data are available on how guidelines are transferred in general and if compliance with guidelines led to better treatment results. A German survey analyzing the physicians' knowledge and their compliance with guidelines for cardiovascular diseases revealed that physicians who adequately knew the guidelines performed essentially not differently from those who did not [7]. For breast cancer, however, recent data suggest a better outcome for patients with guideline-conforming treatment. In this retrospective cohort analysis, more than 3,000 patients were analyzed with reference to guideline violation, resulting in a significant difference with regard to overall and disease-free survival [8]. It seems logical, but there is no evidence that guideline adherence led to a relevant improvement in the prognosis of cancer patients in general, or especially for patients with BTC. More systemic analyses are needed to answer this interesting question.

Another important aspect of this survey was to analyze if there are differences in the treatment and diagnostic procedures according to the affiliation to a specific specialization or the clinical environment. We mainly compared the two most significant subgroups, oncologists $(61.7 \%$ of the respondents) versus gastroenterologists $(27.2 \%)$, postulating that multidisciplinary cooperation is necessary in biliary tract cancer and offers an interesting aspect in the treatment of BTC patients. Differences between gastroenterologists and oncologists could be detected concerning the field of action and the number of treated patients per year. The responding gastroenterologists predominantly indicated to work at university hospitals and to treat more than 25 patients per year, whereas the oncologists in approximately equal parts stated to work in private practice or hospitals and indicated to treat mostly between 11 and 25 patients per year. Noticeable differences could be discerned in the diagnostic use of tumor markers or endoscopic interventions, the application of adjuvant chemotherapy or radiochemotherapy, the application of specific regimens for palliative first-line therapy, and the interval of follow-up. The differences in the clinical environment between these two subgroups may be a confounding factor in this context.

Most answers did not differ considerably so that a consensus can be supposed for most of the relevant diagnostic and therapeutic proceedings among the different disciplines. This may be a positive result of the efforts made in the last decades to standardize and optimize the management of patients with gastrointestinal cancers, by the development of guidelines and by engagement in multidisciplinary tumor boards and professional associations.

The informative value of our survey is limited by the validity of the given answers, which may reflect center-specific procedures. Due to the anonymous evaluation, this aspect could not be further analyzed. A comparison with 'real-life data', as for example by analyzing diagnosis-related group (DRG)associated procedures seems very interesting but difficult to execute. Tumor-specific registers can certainly be considered as another effective and feasible measure in clinical research of daily care practice. Beside clinical studies, they can provide prospective data and evaluate actual daily care practice and clinical outcome, thus correlating study results and patient benefit in real practice. Fortunately, a wide majority of the respondents positively voted for a tumor-specific register; about half of them would participate actively.

It would be interesting to match conclusions from this survey with prospective results from a tumor-specific register. Guideline adherence and potential improvement of clinical outcome may be analyzed by periodical evaluation of real-life practice, as we did with our survey.

\section{Disclosure Statement}

All authors declare no conflicts of interest.

\section{References}

1 Aljiffry M, Walsh MJ, Molinari M: Advances in diagnosis, treatment and palliation of cholangiocarcinoma: 1990-2009. World J Gastroenterol 2009;15:4240-4262.

2 Valle J, Wasan H, Palmer DH, Cunningham D, Anthoney A, Maraveyas A, et al.: Cisplatin plus gemcitabine versus gemcitabine for biliary tract cancer. N Engl J Med 2010;362:1273-1281.

3 Eckel F, Brunner T, Jelic S: Biliary cancer: ESMO clinical practice guidelines for diagnosis, treatment and follow-up. Ann Oncol 2011;22 (suppl 6):vi40-vi44.

4 Boeck S, Bruns CJ, Sargent M, Schafer C, Seufferlein T, Jauch K-W, et al.: Current oncolog- ical treatment of patients with pancreatic cancer in Germany: results from a national survey on behalf of the Arbeitsgemeinschaft Internistische Onkologie and the Chirurgische Arbeitsgemeinschaft Onkologie of the Germany Cancer Society. Oncology 2009;77:40-48.

5 Hassel JC, Kripp M, Al-Batran S, Hofheinz R-D: Treatment of epidermal growth factor receptor antagonist-induced skin rash: results of a survey among German oncologists. Onkologie 2010;33: 94-98.

6 Kikkert WJ, Piek JJ, de Winter RJ, Peters RJ, Henriques JPS: Guideline adherence for antithrombotic therapy in acute coronary syndrome: an overview in Dutch hospitals. Neth Heart J 2010;18:291-299.

7 Karbach U, Schubert I, Hagemeister J Ernstmann N, Pfaff H, Höpp H-W: Physicians' knowledge of and compliance with guidelines: an exploratory study in cardiovascular diseases. Dtsch Ärztebl Int 2011;108:61-69.

8 Wolters R, Wöckel A, Wischnewsky M, Kreienberg R: [Effects of guideline-compliant therapy on the survival of primary breast cancer patients with: results of a retrospective cohort study]. Z Evid Fortbild Qual Gesundhwes 2011;105:468-475. 